\title{
Online rapid sampling microdialysis (rsMD) using enzyme- based electroanalysis for dynamic detection of ischaemia during free flap reconstructive surgery
}

\author{
M. L. Rogers • P. A. Brennan • C. L. Leong • S. A. N. Gowers • \\ T. Aldridge • T. K. Mellor • M. G. Boutelle
}

Received: 30 September 2012 /Revised: 18 January 2013 / Accepted: 20 January 2013 /Published online: 26 February 2013

(C) The Author(s) 2013. This article is published with open access at Springerlink.com

\begin{abstract}
We describe an enzyme-based electroanalysis system for real-time analysis of a clinical microdialysis sampling stream during surgery. Free flap tissue transfer is used widely in reconstructive surgery after resection of tumours or in other situations such as following major trauma. However, there is a risk of flap failure, due to thrombosis in the flap pedicle, leading to tissue ischaemia. Conventional clinical assessment is particularly difficult in such 'buried' flaps where access to the tissue is limited. Rapid sampling microdialysis (rsMD) is an enzyme-based electrochemical detection method, which is particularly suited to monitoring metabolism. This online flow injection system analyses a dialysate flow stream from an implanted microdialysis probe every $30 \mathrm{~s}$ for levels of glucose and lactate. Here, we report its first use in the monitoring of free flap reconstructive surgery, from flap detachment to revascularisation and overnight in the intensive care unit. The on-set of ischaemia by both arterial clamping and failure of venous drainage was seen as an increase in lactate and decrease in glucose levels. Glucose levels returned to normal within 10 min of successful arterial anastomosis, whilst lactate took longer to clear. The use of the lactate/glucose ratio provides a clear predictor of ischaemia on-set and subsequent recovery, as it is insensitive to changes in blood flow such as
\end{abstract}

Published in the topical collection Bioelectroanalysis with guest editors Nicolas Plumeré, Magdalena Gebala, and Wolfgang Schuhmann.

M.L. Rogers and P.A. Brennan have contributed equally to this work.

M. L. Rogers · C. L. Leong · S. A. N. Gowers •

M. G. Boutelle $(\bowtie)$

Department of Bioengineering, Imperial College,

London SW7 2AZ, UK

e-mail: m.boutelle@imperial.ac.uk

P. A. Brennan - T. Aldridge · T. K. Mellor

Maxillofacial Department, Queen Alexandra Hospital,

Portsmouth PO6 3LY, UK those caused by topical vasodilators, like papaverine. The use of storage tubing to preserve the time course of dialysate, when technical difficulties arise, until offline analysis can occur, is also shown. The potential use of rsMD in free flap surgery and tissue monitoring is highly promising.

Keywords Microdialysis - Free tissue transfer - Glucose Lactate $\cdot$ Real time $\cdot$ Ischaemia

\section{Introduction}

Despite the increasing sophistication of modern surgical procedures, complications such as the development of ischaemia due to a disrupted blood supply are relatively common and difficult to detect. If such complications are detected, further emergency surgical intervention may be required. If it goes unnoticed, there is a high associated morbidity.

Microdialysis is a minimally invasive technique ideal for the clinical monitoring of tissue, particularly in difficult to observe areas. In this technique, a thin double-lumen catheter with a semi-permeable membrane at its end is inserted into the tissue. A physiological perfusion fluid is pumped through the catheter using a small pump, and small molecules such as glucose and lactate, pass via the membrane into the fluid creating a dialysate stream. In conventional microdialysis, this dialysate is collected in microvials. One of the main problems with this technique is that, with a perfusion rate of $0.3-2 \mu \mathrm{L} / \mathrm{min}$, the time taken to collect a sufficient volume for assay in a bedside analyser can take $30 \mathrm{~min}$ to an hour. The sample then needs to be introduced into a bedside analyser by hand (requiring attention from busy clinical staff) and the analysis itself usually takes another few minutes before concentrations of glucose, lactate and other metabolites are displayed. This loses dynamic information preventing real-time detection of adverse 
events. An exciting new method for real-time analysis of tissue metabolites has been developed. An online flow injection system, known as rapid sampling microdialysis (rsMD), is used to monitor tissue levels of glucose and lactate at the bedside [1]. rsMD has also successfully been used to monitor metabolites in animal models of ischaemia [2], during human gastrointestinal [3] and human brain surgery [4], as well as monitoring brain injury patients on the intensive care ward [5].

Free tissue transfer is the process of harvesting soft tissue (such as skin and muscle and sometimes with associated bone) together with its blood supply. Healthy tissue is prepared at the donor site and the blood supply is clamped and cut, thus creating a free flap. Microsurgery is then used to re-establish the blood supply at the recipient site. After revascularisation, there is a risk of thrombosis in the artery or vein leading to flap ischaemia and failure. Hence, early recognition of flap compromise is essential for successful reconstruction. The majority of free flaps that fail do so within the first $24 \mathrm{~h} \mathrm{[6]}$ and current clinical assessment includes flap colour, temperature and turgidity. Recently, modalities including Doppler flowmetry and microdialysis for measuring metabolism have gained popularity in predicting flap compromise [7, 8]. However, some question whether these techniques are superior to clinical assessment alone [9]. Conventional microdialysis has already been used to detect tissue ischaemia during free flap surgery [10], in the oral cavity [11] and in buried flaps [12].

Here, we report for the first time the use of real-time microdialysis to monitor free flap surgery and the relocated tissue in the post-operative period.

\section{Materials and methods}

\section{Clinical analysis system}

The dialysate was analysed using rsMD by directly connecting a clinical microdialysis probe (CMA70, MDialysis, Solna Sweden) to an enzyme-based electrochemical detection method via low-volume fluorinated ethylene propylene (FEP) connection tubing $(0.12 \mu \mathrm{L} / \mathrm{cm}$, Thames-Restek, Bucks). An overview of the setup is shown in Fig. 1. The analysis equipment is assembled on a clinically approved isolation trolley (CTL Medical). This is critical as it provides (a) the necessary patient isolation, (b) as importantly acceptability by clinical staff within the operating theatre environment and (c) a workspace for the monitoring team. Patient electrical isolation occurs at three levels: the very high impedance of the saline-filled FEP tubing, electrically isolated power supplies for the potentiostats and overall isolation transformer for the clinical trolley.

The analysis system consists of a custom-made six-port injection valve with two $200 \mathrm{~nL}$ internal sample loops made by Valco Instruments, Switzerland, coupled to a precision HPLC pump (Rheos 2200, Flux Switzerland). Every 30 s, the valve injects microdialysate samples into a mediator flow stream $(1.5 \mathrm{mM}$ ferrocene monocarboxylate, $100 \mathrm{mM}$ sodium citrate, $150 \mathrm{mM}$ sodium chloride, $1 \mathrm{mM}$ EDTA, Kathon (Rohn and Haas), $\mathrm{pH} 7$ ), which passes through either a glucose or a lactate enzyme reactor. The reactors house nitrocellulose-based nanoporous membranes (Millipore) with high enzyme loading for efficient sequential transfer of the electrons from the substrate via hydrogen peroxide to horseradish peroxidase (HRP) which was regenerated by a synthetic mediator, ferrocene monocarboxylate (Sigma-Aldrich) [13]. A downstream electrode ( $3 \mathrm{~mm}$, radial flow glassy carbon disc) operating at $0 \mathrm{~V}$ compared to the $\mathrm{Ag}|\mathrm{AgCl}| \mathrm{Cl}^{-}(150 \mathrm{mM})$ direct reading reference electrode reduces the ferrocene (III) species produced by HRP back to ferrocene (II) to give a current peak; this is shown schematically in Fig. 1b. The use of a reducing potential prevents interference from electroactive species that may be present in the dialysate. Our careful choice of buffer chloride concentration has two benefits: Firstly, our in-house low-noise potentiostats are able to operate using an internally set potential of $0 \mathrm{~V}$, dramatically reducing noise by a factor of 10 [14]. Secondly, it matches the chloride concentration in the microdialysis stream minimising injection artefacts. The potentiostats are connected to a $16 / 35$ Powerlab (ADInstruments) and the data are recorded using LabChart software (ADInstruments) which also timestamps key events during the surgery with the raw data files and allows us to record audio/video files timelocked to the data for unambiguous identification of event timing. The current peak from each sample injection is displayed on a computer screen in real time, with current inverted for convenience (Fig. 1c). This allows the monitoring team to give the surgical team a commentary on glucose and lactate levels during the surgical procedure.

Data analysis

The amplitude of the current peak is related to the concentration of substrate in the dialysate by calibrations. The system is calibrated from 0.25 to $5 \mathrm{mM}$ (four steps each with five replicates) before surgery, after surgery and after monitoring in the ICU by injection of known standards directly into the valve using a glass Hamilton syringe (Fig. 1c, e). This allows accurate conversion of the current signal into concentration. If required, one-off injections of a standard can occur during monitoring to track the assay stability. Typically, the analysis method has 5-day stability and a detection limit of $25 \mu \mathrm{M}$ [15]. The raw data containing injection peaks are processed using MatLab (MathWorks). Purpose-written published algorithms are used to remove common noise artefacts such as spikes, ripples and baseline drift, typically improving the signal-to-noise ratio by $250 \%$ [15]. The peaks are then detected and compared to the 


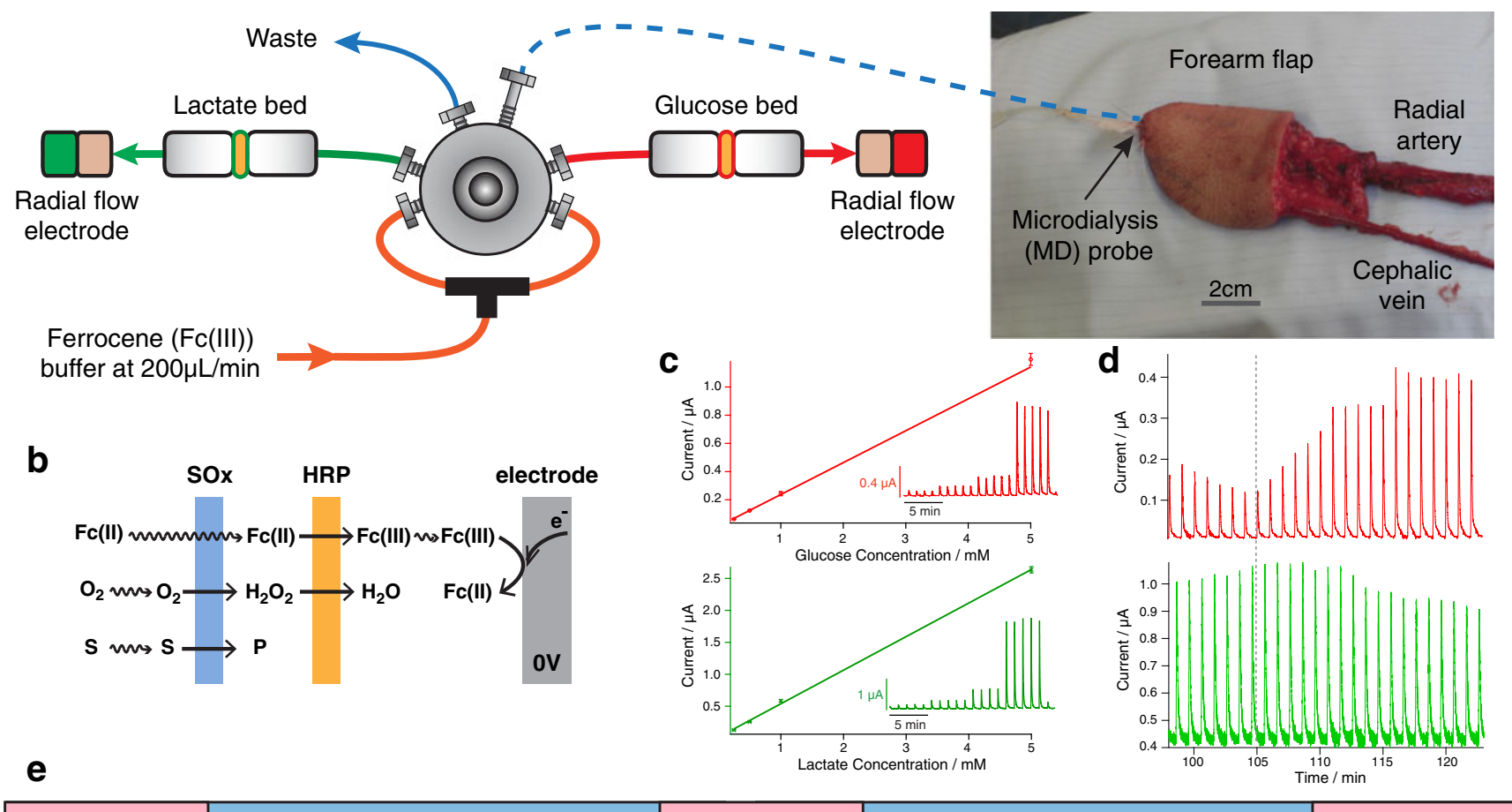

\section{Calibration $\quad$ Surgery (5 - 12 hours)}

Fig. 1 The microdialysis probe is inserted into the tissue flap subcutaneously and sutured into place, shown in the photograph in (a) before the flap has been detached from the blood vessels. The dialysate is introduced to the rsMD injection valve via low-volume FEP connection tubing (blue dotted line). The reaction is mediated by ferrocene monocarboxylate, which is perfused at $200 \mu \mathrm{L} / \mathrm{min}$ using an HPLC pump. The

relevant calibration function to give values for microdialysate concentration.

\section{Storage tubing}

As each surgery is a unique event, the data collected are of high value; hence, an alternative method of data collection has been developed for circumstances when online analysis cannot be achieved. With use of $0.4 \mathrm{~mm}$ ID Portex tubing, $63 \mathrm{~min}$ of dialysate can be collected per $1 \mathrm{~m}$ length at $2 \mu \mathrm{L} / \mathrm{min}$, with retention of temporal information. This allows sections of dialysate to be analysed at a later date. Figure 2 shows the raw data of dialysate collected as the microdialysis probe was removed from the tissue. The dialysate had been stored at $4{ }^{\circ} \mathrm{C}$ for 10 days before analysis, yet we can see stable levels, which fade to zero in both the glucose and lactate graphs at the correct time probe removal.

\section{Sterility in surgery}

One of the main problems was to bridge the sterility barrier from the patient to the analysis system during the often enzyme is loaded onto membranes placed before a glassy carbon electrode which is held at $0 \mathrm{~V}(\mathbf{b})$. The current is recorded as peaks, where amplitude is related to concentration as shown in the calibration curve in (c). Raw data of a successful arterial anastomosis (indicated by the dotted grey line) are shown in (d). A time line indicating the calibration protocol throughout monitoring is shown in (e)

lengthy periods of surgery. The microdialysis probe and perfusion solution are both sterile, however, our analysis system and connection tubing are not. To overcome this, we use a sterilised tubular polyethylene camera drape. This allows the non-sterile equipment (CMA microdialysis pump and connection tubing) to be placed inside the drape and close to the patient without the worry of contamination.

\section{Surgical protocol}

Ablative surgery, to remove the tumour, was completed and the flap was raised concurrently. Prior to division of the blood vessels (creating a free flap), a CMA70 microdialysis probe (MDialysis, Solna, Sweden, $10 \mathrm{~mm}$ membrane, $20 \mathrm{kDa}$ cut-off) was inserted and secured subcutaneously into the flap using a suture at the hard plastic union (avoiding the soft concentric tubes); a photograph can be seen in Fig. 1a. The probe was perfused with sterile T1 solution (MDialysis) at $2 \mu \mathrm{L} / \mathrm{min}$. Prior to detachment of the flap, baseline dialysate levels of glucose and lactate were recorded. A vasodilator, papaverine, was then applied topically to the blood vessels, increasing blood flow to the 
Fig. 2 Raw data from dialysate collected during surgery into a collection tubing. The probe was removed from the tissue during collection. The dialysate was stored for 10 days at $4{ }^{\circ} \mathrm{C}$ and analysed using rsMD. The data shown have had no noise removal algorithms applied.

The top graph shows glucose data and the bottom graph shows lactate data
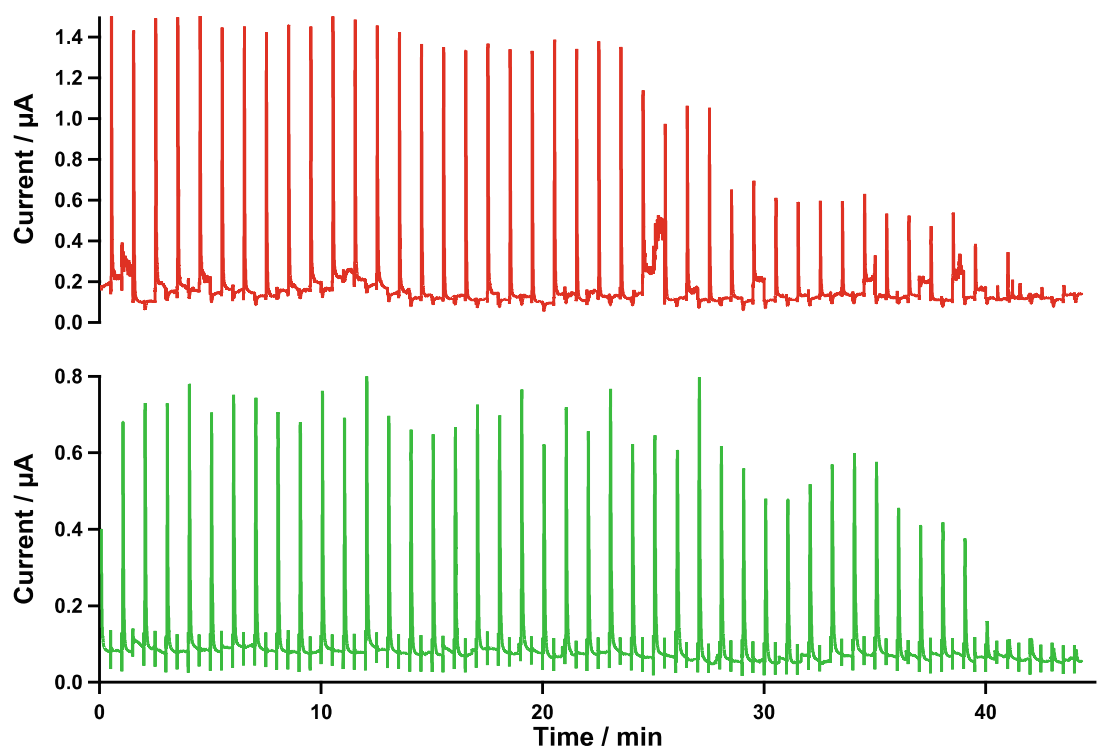

tissue. The microdialysis probe remained connected with online analysis as the flap was detached and moved to the donor site. The free flap artery was anastomosed to the facial artery first, followed by the vein and then reconstruction of the recipient site. The patients were transferred to the ICU and kept sedated and ventilated overnight. rsMD was continued for a 10 -h period before the microdialysis probe was removed. The glucose and lactate levels were recorded, with routine flap observations (colour, temperature and turgidity) carried out in addition.

\section{Results and discussion}

Of the five cases monitored to date, two cases will be described in detail, highlighting key events during surgery.
Case study 1

The glucose and lactate levels throughout surgery of a 39year-old male presenting a carcinoma of the tongue are shown in Fig. 3. Ten minutes after probe insertion, baseline dialysate levels stabilised to $1.26 \pm 0.03 \mathrm{mM}$ glucose and $0.57 \pm 0.02 \mathrm{mM}$ lactate $(n=10)$. These values are lower than the better-perfused bowel $(2.77 \pm 0.57 \mathrm{mM}$ glucose, $0.77 \pm$ $0.17 \mathrm{mM}$ lactate) [3] but much higher than the brain (glucose typically ranges from 0.3 to $0.7 \mathrm{mM}$ and lactate baseline levels range from 0.4 to $0.7 \mathrm{mM}$ ) [5] where blood flow is tightly regulated.

Before detachment of the flap from the forearm, a vasodilator, papaverine, was applied to the blood vessels. After application, the levels of both glucose and lactate increased. Most probably, this is due to an increase in the blood
Fig. 3 Analysis of data collected during free flap surgery. The top graph (blue) shows the lactate/glucose ratio. The bottom graph shows glucose (red) and lactate (green) levels throughout the reconstructive surgery. Time zero is the time from stable baseline (10 min after probe insertion). The dotted lines indicate key events logged as communicated by the surgeons to the monitoring team in real time

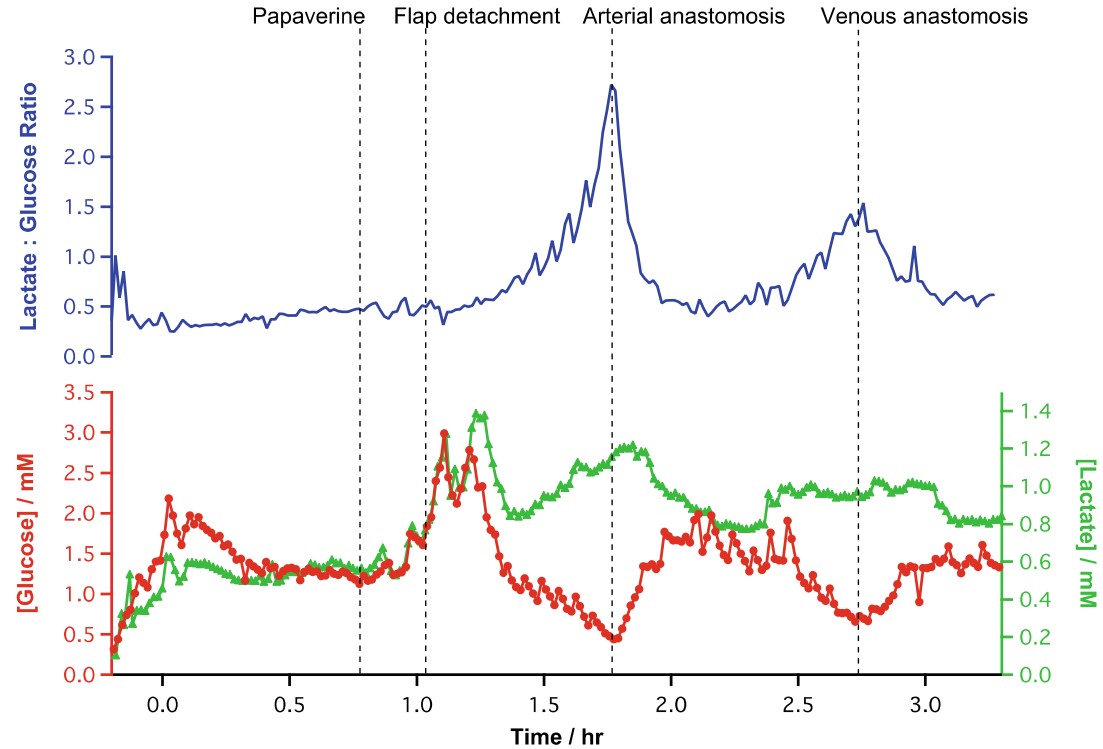


volume to the tissue that the probe is sampling from. As the changes of glucose and lactate are in the same direction, they could be due to a transient change in the efficiency of microdialysis probe recovery [1]. To control for this, and hence simplify the analytical measurement, a ratio of the two metabolites has been used. The lactate/glucose ratio does not change during the application of papaverine, indicating the same percentage increase in both lactate and glucose reflecting a change in probe recovery.

When the flap was detached, by division of the blood vessels (just after $1 \mathrm{~h}$ in Fig. 3), the dialysate glucose concentration fell and the lactate concentration increased. This change in levels in opposite directions is a reliable microdialysis signature [1]. This is reflected in the near exponential rise in the lactate/glucose ratio seen after the artery has been clamped and severed.

Upon successful arterial anastomosis (reconnection of the arterial supply to the free flap in its transferred position in the oral cavity, at $1.8 \mathrm{~h}$ in Fig. 3), the arterial clamp is released and a check is made in any leak at the anastomosis site and that blood visibly flows into the flap and flows out of the veins. Once blood flow is reconfirmed, all the clamps are re-applied. We observed a rapid rise in dialysate glucose concentration to $1.75 \pm 0.14 \mathrm{mM}(n=10)$, within $11 \mathrm{~min}$. The raw data for this event are shown in Fig. 1d. The lactate level was maintained for $7 \mathrm{~min}$ before returning to an elevated baseline $(0.78 \pm 0.01 \mathrm{mM}, n=10)$. This may represent the fact that lactate is neither being utilised nor cleared efficiently by local blood flow. Although levels return to near baseline, signs of a second ischaemia developed within $30 \mathrm{~min}$, albeit the concentration changes of glucose and lactate were not as large. This is most clearly seen in the lactate/glucose ratio.

Once the venous anastomosis has been completed (at $2.75 \mathrm{~h}$ in Fig. 3), all of the clamps are removed and the second ischaemia is relieved, seen as a fall in lactate/glucose ratio, a product of the sharp rise in glucose and delayed fall in lactate dialysate levels. Therefore, we conclude that a ratiometric approach to monitoring tissue ischaemia gives a strong indicator of flap health compared to the evaluation of individual metabolites. Furthermore, the excellent signal to noise of the lactate/glucose ratio makes it a robust predictor of the on-set of ischaemia that is suitable for real-time clinical use.

The patient was transferred to ICU where monitoring was continued (Fig. 4). The levels of both glucose and lactate are higher at the beginning of this monitoring period than at the end of surgery. However, there is a clear trend of falling lactate concentration and rising glucose levels. Over the $10 \mathrm{~h}$ that the flap was monitored in the ICU, the lactate/glucose ratio was seen to steadily fall to the level it was before the flap was raised from the forearm. The tissue reconstruction was successful by normal clinical indicators.

\section{Case study 2}

Figure 5 shows two extracts of glucose and lactate levels and the ratio of a more complicated surgery of a 47 -year-old male, where muscle and bone from the leg were used to reconstruct the jaw. Baseline levels of glucose and lactate were $2.05 \pm 0.36$ $(n=10)$ and $5.55 \pm 0.60 \mathrm{mM}(n=10)$, respectively.

As with case study 1 , after detachment of the flap, the tissue concentration of glucose fell and the lactate/glucose ratio increased indicating tissue ischaemia. Upon the first attempt at anastomosis (both arterial and venous), the changes in the level of glucose were much smaller than that of case study 1 . The levels of glucose and lactate remain on their downward and upward trends, respectively, which is more clearly shown by a continuation of an upward trend in the lactate/glucose ratio as shown in Fig. 5a. This demonstrates the unsuccessful reperfusion of blood through the free flap tissue. Upon investigation,
Fig. 4 Analysis of data collected during ICU monitoring. The top graph shows the lactate/glucose ratio. The bottom graph shows the glucose (red) and lactate (green) levels
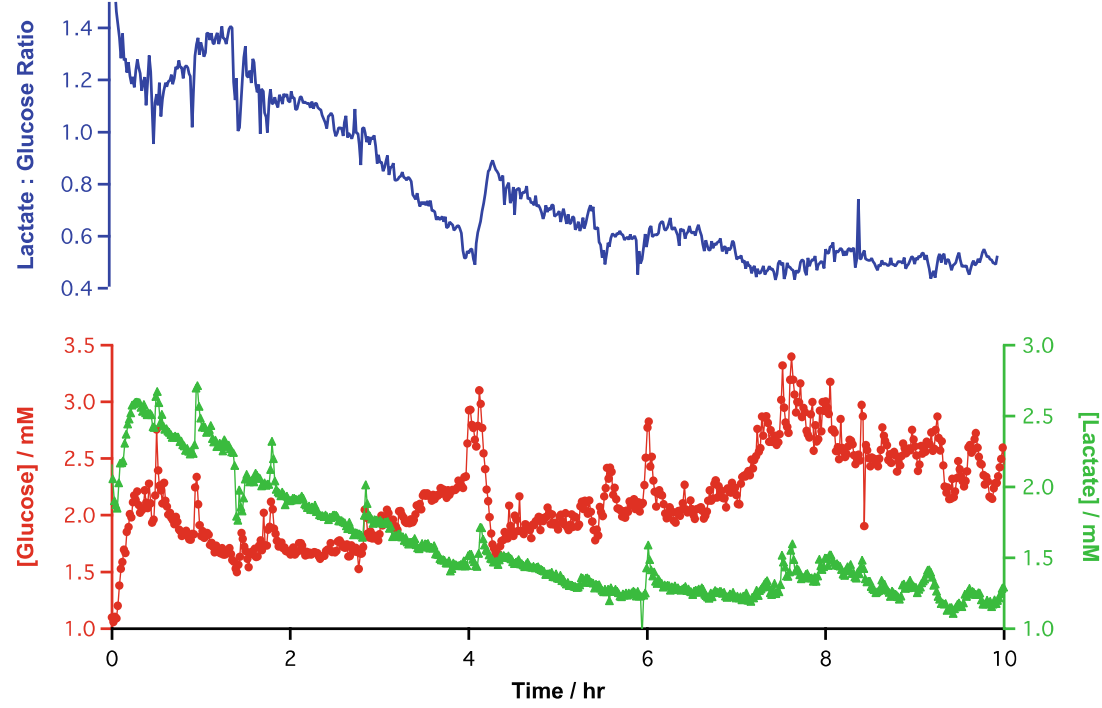
Fig. 5 Two extracts from one surgery showing (a) a failed anastomosis and (b) a

successful anastomosis in terms of lactate (green), glucose (red) and the lactate/glucose ratio (blue). Time zero is the time of connection, conveyed by the surgeons and recorded in real time a
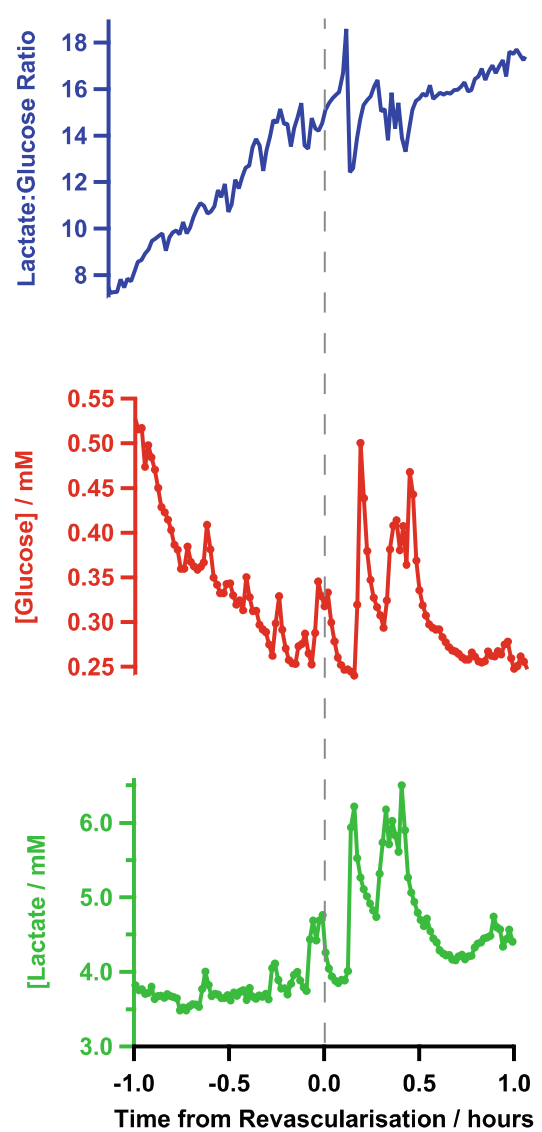

b
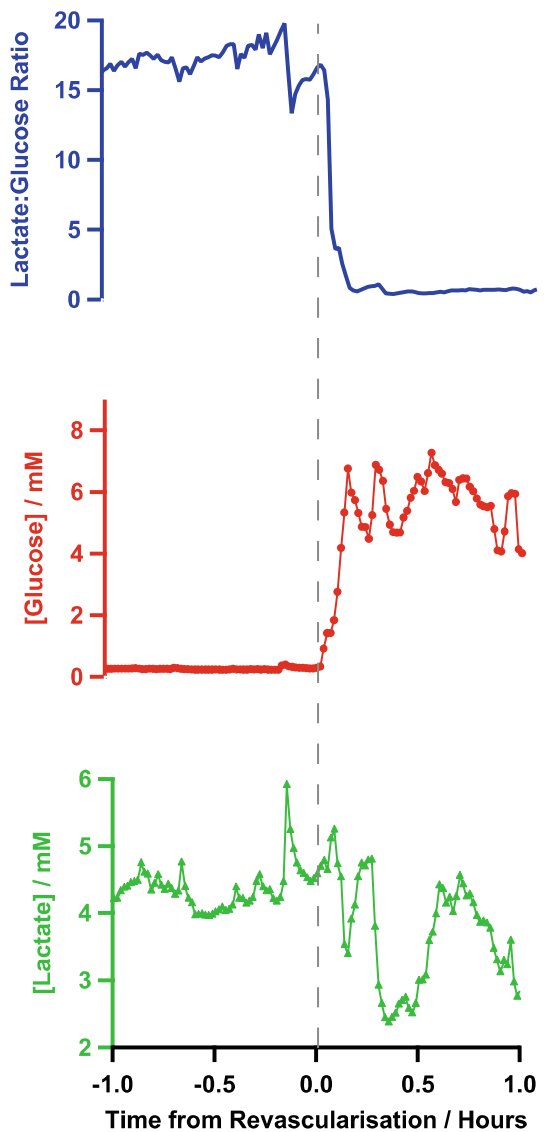

large blood clots were found at all anastomosis sites and later an undetected rare blood disorder was confirmed.

The patient was then thrombolysed and the anastomosis was re-attempted with success (shown in Fig. 5b). Here, the glucose concentration dramatically increases from $0.32 \pm$ $0.02 \mathrm{mM}(n=10)$ to $4.85 \pm 0.48 \mathrm{mM}(n=10)$ whilst the lactate levels are slower to change. The successful reperfusion is

Fig. 6 Histograms highlighting key moments during rsMD monitoring: (i) Baseline. (ii) Flap detached. (iii) Flap reattached. (iv) Levels at the end of monitoring in ICU. A Mann-Whitney-Wilcoxon test was used to assess the changes from baseline within each case a

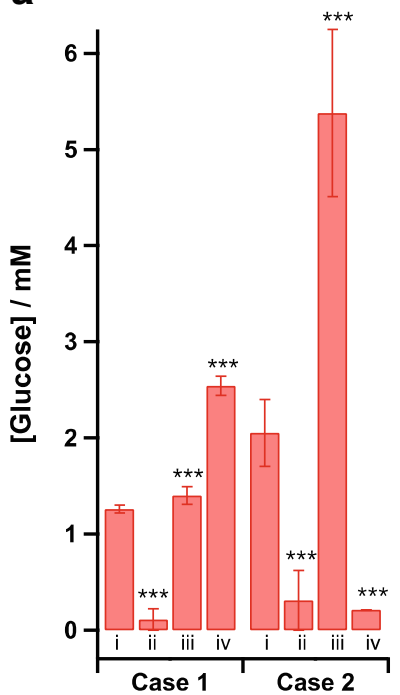

shown most clearly in the lactate/glucose ratio, which falls dramatically from $17.19 \pm 0.44(n=10)$ to $0.77 \pm 0.07(n=10)$. These data highlight the reliability of the monitoring system for online and real-time detection of successful reperfusion during surgery.

Once the patient was transferred to ICU, monitoring continued for $10 \mathrm{~h}$. During this time, the level of glucose dropped

b

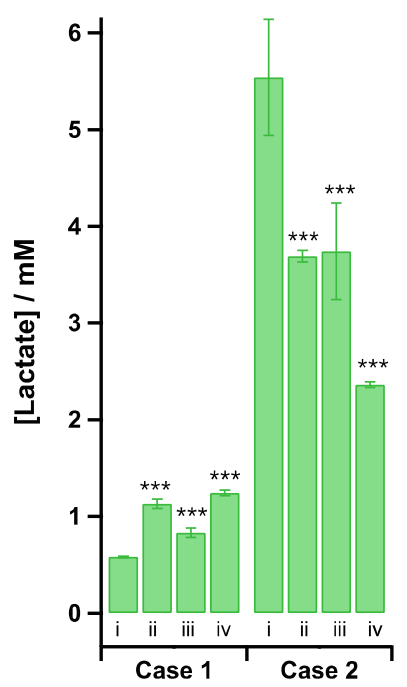

C

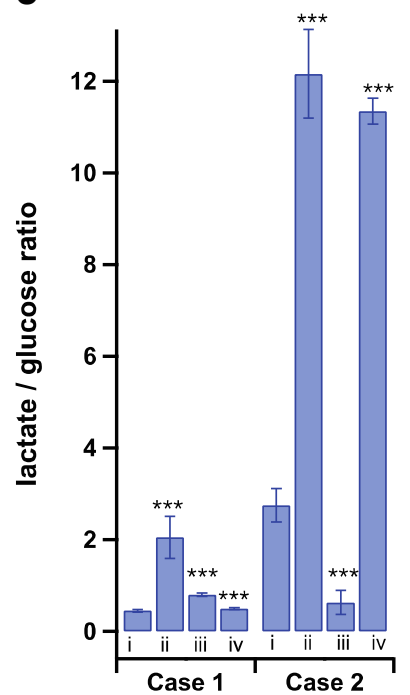


from $4.41 \pm 0.08 \mathrm{mM}(n=10)$ to $0.21 \pm 0.002 \mathrm{mM}(n=10)$ and the lactate/glucose ratio gradually increased from $1.10 \pm 0.02$ $(n=10)$ to $11.44 \pm 0.26(n=10)$. Some time after microdialysis monitoring was ceased, the flap failed, and at this later date, further surgical intervention was required.

An overview of the dialysate levels of glucose and lactate and the lactate/glucose ratio throughout these two patient cases is shown in Fig. 6. A Mann-Whitney-Wilcoxon test has tested for changes from the baseline within each case (before the blood supply to the tissue had been cut) to three clear points throughout the monitoring period, detachment of the flap, full successful reconnection and the levels in ICU. All changes show high significance indicating that monitoring these key metabolic markers during free flap and reconstructive surgery has potential to evaluate the 'health' of the tissue in real time. In both cases when the blood supply to the flap is disconnected, the lactate/glucose ratio clearly increases. This change is much harder to see from assessment of the glucose and lactate data taken individually, although the levels of glucose in the dialysate seem to be more responsive to, and therefore more indicative of, tissue perfusion. When the tissue has successfully been reconnected, again it is clear to see the trends in both the lactate/glucose ratio and the level of dialysate glucose reverse. In ICU, case 1 shows no complications, with a higher level of dialysate glucose than the baseline and a low lactate/glucose ratio. Whilst at the end of monitoring of case 1, the flap was successful, and few days after microdialysis monitoring ceased, the flap failed and further surgery was required. This may explain the low dialysate glucose levels and high lactate/glucose ratio seen.

\section{Conclusion}

We have successfully monitored the metabolic health of a free flap, in real time, during and after free flap reconstructive surgery, using rsMD. The potential value of these measurements to reconstructive surgeons is exciting. rsMD offers a considerable advantage over offline microdialysis because of the inherent time delays that necessarily occur with the latter technique. Using a lactate/glucose ratiometric approach is a strong indicator of tissue health, as it is insensitive to flow and recovery artefacts, leaving a clear and easily interpretable predictor of ischaemia. In the exemplar cases, we can clearly see ischaemia on-set following both arterial occlusion and failure of venous drainage, the two most common causes of flap failure. Unsuccessful anastomosis during surgery is shown to be easily distinguishable to that of successful anastomosis and tissue reperfusion. We have also demonstrated, in real time, the effects of topically applied drugs (papaverine). The research applications for further study of the real-time metabolic changes that occur during free flap harvest, detachment and re-vascularisation are many.
From the patients monitored to date, there is considerable variability in the tissue levels and responses between patients due to many co-variates such as age, sex, underlying health issues, etc. as well as effectiveness of the microdialysis sampling. Full statistical analysis will require a larger cohort. From these preliminary data, the relative changes in glucose, lactate and particularly the lactate/glucose ratio within a patient data set clearly show dynamic trends that reflect tissue health.

Future work includes monitoring a larger cohort of patients for full clinical analysis. On-going optimisation using new technologies, such as a microfluidic manifold with incorporated electrochemical sensors [16], will only improve the system and the quality of the results further still.

Acknowledgments We are grateful to Mr. Paul Collinson and Prospect Diagnostics, Derby, UK for supplying the microdialysis catheters. We thank the EPSRC, Imperial BRC, BBSRC and the Wellcome Trust for funding.

Open Access This article is distributed under the terms of the Creative Commons Attribution License which permits any use, distribution, and reproduction in any medium, provided the original author(s) and the source are credited.

\section{References}

1. Parkin MC, Hopwood SE, Boutelle MG, Strong AJ (2003) Resolving dynamic changes in brain metabolism using biosensors and on-line microdialysis. Trend Analy Chem 22:487-497

2. Hashemi P, Bhatia R, Nakamura H et al (2009) Persisting depletion of brain glucose following cortical spreading depression, despite apparent hyperaemia: evidence for risk of an adverse effect of Leo's spreading depression. J Cereb Blood Flow Metab 29:166-75

3. Deeba S, Corcoles EP, Hanna GB et al (2008) Use of rapid sampling microdialysis for intra-operative monitoring of bowel ischemia. Dis Colon Rectum 5:1408-13

4. Bhatia R, Hashemi P, Razzaq A et al (2006) Application of rapidsampling, online microdialysis to the monitoring of brain metabolism during aneurysm surgery. Neurosurg 58:313-20

5. Feuerstein D, Manning A, Hashemi P et al (2010) Dynamic metabolic response to multiple spreading depolarizations in patients with acute brain injury: an online microdialysis study. J Cereb Blood Flow Metab 30:1343-55

6. Chen KT, Mardini S, Chuang DC et al (2007) Timing of presentation of the first signs of vascular compromise dictates the salvage outcome of free flap transfers. Plast Reconstr Surg 120:187-95

7. Abdel-Galil K, Mitchell D (2009) Postoperative monitoring of microsurgical free tissue transfers for head and neck reconstruction: a systematic review of current techniques. Part I. Noninvasive techniques. Br J Oral Maxillofac Surg 47:3515

8. Abdel-Galil K, Mitchell D (2009) Postoperative monitoring of microsurgical free-tissue transfers for head and neck reconstruction: a systematic review of current techniques. Part II. Invasive techniques. Br J Oral Maxillofac Surg 47:43842

9. Whitaker IS, Rozen WM, Chubb D et al (2010) Postoperative monitoring of free flaps in autologous breast reconstruction: a multicenter comparison of 398 flaps using clinical monitoring, 
microdialysis, and the implantable Doppler probe. J Reconstr Microsurg 26:409-16

10. Birke-Sorensen H, Andersen NT (2010) Metabolic markers obtained by microdialysis can detect secondary intestinal ischemia: an experimental study of ischemia in porcine intestinal segments. World J Surg 34:923-32

11. Nielsen HT, Gutberg N, Birke-Sorensen H (2011) Monitoring of intra-oral free flaps with microdialysis. Br J Oral Maxillofac Surg 49:521-6

12. Mourouzis C, Anand R, Bowden JR, Brennan PA (2007) Microdialysis: use in the assessment of a buried bone-only fibular free flap. Plast Reconstr Surg 120:1363-6
13. Cass AE, Davis G, Francis GD et al (1984) Ferrocene-mediated enzyme electrode for amperometric determination of glucose. Anal Chem 56:667-71

14. Feuerstein D (2009) Development and use of bioanalytical instrumentation and signal analysis methods for rapid sampling microdialysis monitoring of neuro-intensive care patients, $\mathrm{PhD}$ Thesis

15. Feuerstein D, Parker KH, Boutelle MG (2009) Practical methods for noise removal: application to spikes, nonstationary quasiperiodic noise and baseline drift. Anal Chem 12:4987-4994

16. Rogers M, Leong C, Niu X, de Mello A, Parker KH, Boutelle MG (2011) Optimisation of a microfluidic analysis chamber for the placement of microelectrodes. Phys Chem Chem Phys 13:5298-303 